\title{
Factores fisicoquímicos que regulan la motilidad espermática en peces: aspectos básicos y aplicados. Una revisión
}

\author{
Physical-chemical factors that regulate spermatic motility in fish: \\ basic and applied aspects. A review \\ I Valdebenito*, C Fletcher, V Vera, J Fernández \\ Escuela de Acuicultura, Universidad Católica de Temuco, Temuco, Chile.
}

\begin{abstract}
SUMMARY
In most teleost fish with external fertilization, the spermatozoids are immobile in the seminal fluid and they are activated only after making contact with an aqueous medium. The time of flagellar movement is short in most species. Furthermore, the factors that trigger this process are different for each one. However, almost for all species, factors such as osmolarity, temperature, $\mathrm{pH}$ and ionic composition have a key role in activating or modulating the flagellar activity. The present review examines the factors involved in the activation of sperm and its regulation in fish with external fertilization. Additionally, an analysis is conducted regarding the management of commercially farmed fish semen, with emphasis on salmonid species.
\end{abstract}

Palabras clave: peces, reproducción, salmónidos, motilidad espermática.

Key words: fish, reproduction, salmonid, sperm motility.

\section{INTRODUCCIÓN}

En la actualidad, las actividades piscícolas se basan en la capacidad de producir las nuevas generaciones de especímenes de alta calidad biológica ("semillas") para iniciar una nueva fase de engorda en forma exitosa. Para esto es fundamental manejar adecuadamente los reproductores y el producto obtenido de ellos: los gametos. Estas células presentan características muy particulares (haploides y de rápido envejecimiento, por ejemplo) que las hacen merecedoras de la "mayor atención" por parte de quien las manipula, ya que se encuentran muy cerca de "su muerte".

En muchas pisciculturas, frecuentemente esto no ocurre, debiendo los gametos "soportar" prolongados almacenamientos o transportes que no les otorgan las condiciones fisicoquímicas que necesitan para sobrevivir adecuadamente y luego generar un encuentro de gametos masculinos y femeninos exitoso que permita a los productores obtener altos porcentajes de fecundación.

El objetivo de esta revisión es analizar los factores que regulan o alteran la motilidad espermática en peces "de fecundación externa" antes y después del contacto con el agua para dar a conocer herramientas que optimicen las metodologías utilizadas en piscicultura y así mejorar la capacidad fecundante de los espermatozoides.

Aceptado: 21.01.2009.

* Casilla 15-D Temuco, Chile, ivisler@uct.cl.

\section{ANTECEDENTES GENERALES}

La mayoría de los espermatozoides de peces teleósteos presentan una estructura primitiva y carecen de acrosoma, por lo que fecundan los oocitos penetrando a través del micropilo, una pequeña abertura (o varias, dependiendo de la especie) en las membranas del oocito que permite el contacto definitivo de las membranas de ambos gametos (Rudolfsen y col 2008). En salmón sockeye, la fecundación ocurre en el 25\% de los huevos durante los primeros $0,5 \mathrm{~s}$ posteriores a la mezcla de los gametos y luego de $5 \mathrm{~s}$ ya se ha fecundado el $80 \%$ de los huevos (Hoysak y Liley 2001).

En peces teleósteos de fecundación externa, los espermatozoides se encuentran inmóviles en el tracto genital del macho (cuadro 1) y son activados sólo después de tomar contacto con el medio acuoso (cuadro 2), ya sea en agua dulce, salobre y/o fluido ovárico (Ginzburg 1972, Scott y Baynes 1980, Billard y Cosson 1986, Billard y Cosson 1989, Goodall y col 1989, Levanduski y Cloud 1988, Billard y col 1993, Morisawa 1994, Billard y col 1995, Krasznai y col 1995, Ohta y col 1997, Paniagua-Chávez y col 1998, Woolsey y col 2006, Rudolfsen y col 2008). Regularmente, en cada especie varían: 1) los factores que activan la motilidad espermática, 2) la duración de ésta y 3) el patrón de actividad flagelar, lo que hace difícil transferir metodologías o protocolos de manejo de una especie a otra en forma exitosa.

Los factores físicos, químicos y fisiológicos que activan la motilidad del espermatozoide en peces son variados, dependiendo de la especie y del medio donde habita. Entre los factores (o interacción entre algunos de ellos) 
Cuadro 1. Factores que bloquean la motilidad espermática en peces de fecundación externa. Factors that block sperm motility in fish with external fertilization.

\begin{tabular}{llccccccc}
\hline Especie/Grupo & \multicolumn{1}{c}{ N. Científico } & Sucrosa & $\mathrm{K}+$ & $\mathrm{T}^{\mathrm{o}}$ & Osmolaridad & $\mathrm{pH}$ & Urea & Fuente \\
\hline Salmonidae & & $\uparrow$ & $\uparrow$ & - & $\uparrow$ & $\downarrow$ & - & 1,2 y 3 \\
Ciprinidae & & - & - & - & $\uparrow$ & - & $\uparrow$ & 4 \\
Acipenseridae & & - & $\uparrow$ & - & $\uparrow$ & - & - & 1 \\
Pez gato canal & Ictalurus punctatus & - & - & - & $\uparrow$ & - & - & 5 \\
Burbot & Lota lota maculosa & - & $\uparrow$ & $\uparrow$ & $\uparrow$ & $\downarrow$ & - & 1 \\
Perca & Perca fluviatilis & - & - & - & $\uparrow$ & - & - & 6 \\
Pejerrey & Odontesthes & - & - & - & $\downarrow$ & - & - & 7 \\
& bonariensis & & & & & & & \\
Puye & Galaxias maculatus & - & - & - & $\uparrow$ & - & - & 8 \\
Lamprea marina & Petromyzon marinus & - & - & - & $\downarrow$ & - & - & 9 \\
Marine puffer & Fugu niphobles & - & - & - & $\uparrow$ & - & - & 4 \\
Bacalao & Gadus morhua & - & - & - & $\uparrow$ & - & - & 4 \\
\hline
\end{tabular}

- : Sin información

$\uparrow:$ : Aumento

$\downarrow$ : Disminución

1. Zuccarelli y col 2007, 2. Morisawa y col 1993, 3. Billard 1988, 4. Cosson y col 1999, 5. Billard y col 1997, 6. Lahnsteiner y col 1995, 7. Strussman y col 1994, 8. Valdebenito 2004, 9. Ciereszko y Dabrowski 2002.

Cuadro 2. Factores que activan la motilidad espermática en peces de fecundación externa. Factors that trigger sperm motility in fish with external fertilization.

\begin{tabular}{|c|c|c|c|c|c|c|c|c|c|}
\hline Especie/Grupo & N. Científico & $\mathrm{Ca}^{++}$ & $\mathrm{K}^{+}$ & FIME $^{1}$ & Osmolaridad & $\mathrm{pH}$ & $\mathrm{FP}^{2}$ & AMPc & Fuente \\
\hline Salmonidae & & $\uparrow$ & - & - & $\downarrow$ & 8,8 & $\uparrow$ & $\uparrow$ & 1 \\
\hline Ciprinidae & & - & - & - & $\downarrow$ & $6-9$ & - & - & 1 у 2 \\
\hline Acipenseridae & & - & - & - & $\downarrow$ & - & - & - & 1 \\
\hline Pez gato africano & $\begin{array}{l}\text { Pangasius } \\
\text { hypophthalmus } \\
\text { P. gigas }\end{array}$ & - & - & - & $\downarrow$ & - & - & - & 3 \\
\hline Pez gato canal & Ictalurus punctatus & - & - & - & $\downarrow$ & - & - & - & 4 \\
\hline Perca & Perca fluviatilis & - & - & - & $\downarrow$ & $7-8$ & - & - & 5 \\
\hline Puye & Galaxias maculatus & - & - & - & $\downarrow$ & - & - & - & 6 \\
\hline Pejerrey & $\begin{array}{l}\text { Odontesthes } \\
\text { bonariensis }\end{array}$ & - & $\uparrow$ & - & $\uparrow$ & - & - & - & 7 \\
\hline Turbot & $\begin{array}{l}\text { Scophthalmus } \\
\text { maximus }\end{array}$ & - & - & - & $\uparrow$ & 9 & - & - & 1 y 8 \\
\hline Halibut & Hippoglossus $h$. & - & - & - & $\uparrow$ & 9 & - & - & 1 у 9 \\
\hline Sea bream & Sparus auratus & - & - & - & $\uparrow$ & - & - & - & 10 \\
\hline Sea bass & Dicentrarchus labrax & - & - & - & $\uparrow$ & 9 & - & - & 1 у 10 \\
\hline Herring & Clupea palasii & $\uparrow$ & - & $\uparrow$ & $\uparrow$ & - & - & - & 11 \\
\hline Anguilla japonesa & Anguilla japonica & - & $\uparrow$ & - & $\uparrow$ & - & - & - & 12 у 13 \\
\hline Paddlefish & Polyodon spathula & $\uparrow$ & $\downarrow$ & - & - & - & - & - & 1 \\
\hline Esturión & Acipenser baeri & $\uparrow$ & - & - & - & - & - & - & 1 \\
\hline
\end{tabular}

FIME: Factor Iniciador de la Motilidad Espermática

Fosforilación de proteínas

- : Sin información

$\uparrow:$ : Aumento

$\downarrow \quad$ : Disminución

1. Cosson y col 1999; 2. Billard y col 1995; 3. Mongkonpunya y col 1996; 4. Bates y col 1996; 5. Lahnsteiner y col 1995; 6. Valdebenito $2004 ; 7$. Strussman y col 1994; 8. Geffen y Frayer, 1993; 9. Billard y col 1993; 10. Billard, 1978; 11. Morisawa, 1994; 12. Ohta y Izawa, 1996; 13. Ohta y col 1997. 
que desencadenan la actividad flagelar se encuentran los cambios en la presión osmótica como en la carpa, pez gato y merluza (Morisawa y col 1983, Goodall y col 1989, Billard $1990^{\mathrm{a}}$, Billard y col 1993, 1995 b, Wayman y col 1996, Wayman y Tiersch 1998), en la composición iónica (trucha, salmón, arenque) (Billard y Cosson 1989, Lahnsteiner y col 1993, Otha y col 1997), en el pH (Márian y col 1997, Billard y Cosson 1986, Billard y Cosson 1989, Krasznai y col 1995) e incluso el contacto con la orina en el caso del pejerrey (Strüssmann y col 1994, Billard y col 1995 , Billard y Cosson 1997).

Según Morisawa y Suzuki (1980), existirían cuatro modelos básicos que regulan la motilidad en espermatozoides de teleósteos:

1) un medio hipotónico activa la motilidad de espermatozoides de ciprínidos dulceacuícolas,

2) un medio hipertónico induce la motilidad de los espermatozoides en peces marinos,

3) una reducción en $\mathrm{K}^{+}$activa los espermatozoides de salmonídeos y

4) la presencia de cationes (especialmente $\mathrm{K}^{+}$) induce la actividad de espermatozoides de peces vivíparos.

Linhart y col (1995) señalan que la presión osmótica, concentración de potasio y sucrosa y un $\mathrm{pH}$ menor a 7 del plasma seminal, son los principales factores que inhiben la motilidad del espermatozoide en salmónidos y que la presión osmótica parece ser el mayor inhibidor en ciprínidos.

En la mayoría de las especies que presentan fecundación externa, el espermatozoide mantiene por un corto periodo de tiempo la motilidad una vez que ha tomado contacto con el medio externo (Ginzburg 1972, Morisawa 1994). Esto es, producto del escaso número de mitocondrias (1 a 5, frecuentemente) que presenta el espermatozoide en su pieza media, formando un anillo mitocondrial, lo que reduce rápidamente los niveles de ATP intracelular (Billard y Cosson 1986). Se ha observado que el periodo de actividad flagelar de los espermatozoides es mucho más largo en peces que desovan en agua salada que en los que desovan en agua dulce (Ginzburg 1972, Billard 1990b, Billard y col 1995 b). Así por ejemplo, en Oncorhynchus mykiss la actividad flagelar del espermatozoide es de aproximadamente 30 segundos (Billard 1990); en Salmo salar es de 176 segundos (Ginzburg 1972), en Dicentrarchus labrax de 180 segundos (Billard y col 1997), en Huso huso de 14 a 15 minutos y en Capialosa volgensis de 30 a 40 minutos (Ginzburg 1972). Sin embargo, el tiempo de motilidad del espermatozoide depende de diferentes factores. Billard y Cosson (1986) señalan que el tiempo de motilidad del espermatozoide de peces es altamente dependiente de la temperatura y del pH del medio de dilución; a baja temperatura el espermatozoide es menos activo que a temperaturas más altas, pero la duración total del tiempo de movimiento es mayor (Billard y Cosson 1986, Alavi y
Cosson 2006). Ginzburg (1972) señala que el periodo de motilidad puede ser también afectado por otras variables, particularmente por la concentración de espermatozoides en suspensión; a una mayor concentración se observa un menor tiempo de motilidad, esto debido a cambios en la presión parcial de oxígeno y $\mathrm{CO}_{2}$ y del $\mathrm{pH}$ en el medio.

En salmónidos, después de 15 min de la activación, los niveles de ATP retornan a sus niveles iniciales, por lo que al ser nuevamente diluidos en un medio activante alcanzan a una "segunda fase de dilución" con características similares a la primera fase (Alavi y Cosson 2006).

La motilidad del espermatozoide es considerada como uno de los parámetros más utilizados para determinar la calidad del semen. Levanduski y Cloud (1988), Terner (1986), Billard (1988), Redondo-Müller y col (1991 en Lin y Dabrowski 1996), Trippel y col (1991), Zavos y col (1996), Woolsey y col (2006) y Rudolfsen y col (2008), entre otros autores, señalan que la motilidad es el mejor indicador de la capacidad fecundante frente a la duda de la calidad del semen, además de ser un parámetro de fácil evaluación mediante el uso de un microscopio óptico y de escalas preestablecidas tales como la de SánchezRodríguez y Billard (1977), la de Aas y col (1991) y Estay y col (1994). En los últimos años se utilizan sistemas de análisis de imágenes como el NIH Image (Bates y Tiersch 1997) o el CASA (Computer Assisted Sperm Analysis) que permiten realizar una evaluación más objetiva de este parámetro (Ciereszko y col 1996, Cosson y col 1999, Elofsson y col 2003, Alavi y Cosson 2005).

\section{FACTORES QUE REGULAN LA MOTILIDAD ESPERMÁTICA EN PECES}

En peces ovíparos, la fecundación externa está sometida al estrés del medio ambiente. La manipulación del semen in vitro para almacenaje y fecundación requiere la determinación de las condiciones óptimas de densidad espermática, de temperatura, oxígeno, dióxido de carbono, protones $(\mathrm{pH})$, osmolaridad, cationes y sustratos metabolizables para la iniciación y mantención de la motilidad (Goodall y col 1989, Billard y col 1995a, Cosson y col 1999, Rudolfsen y col 2008). Algunos de los principales factores que regulan la motilidad espermática son:

\section{OSMOLARIDAD}

Todos los organismos acuáticos se enfrentan con problemas osmóticos en alguna etapa de su ciclo de vida. Por esto han desarrollado una variedad de mecanismos que emplean para mantener las concentraciones osmóticas internas apropiadas y prevenir el desarrollo de presiones osmóticas que puedan provocar problemas. La osmorregulación implica el mantenimiento de una concentración osmótica interna diferente de la del medio, además de la regulación de la composición y de las concentraciones 
iónicas en diversos compartimientos para asegurar el funcionamiento correcto de las células y tejidos.

Osmorregulación, se refiere a los procesos relacionados con la regulación de la presión osmótica y la concentración de sales. Estos procesos han tenido un efecto importante en la especialización y diversificación de las especies acuáticas a lo largo de la evolución.

Varios estudios han enfatizado los efectos de la osmolaridad en la motilidad espermática en teleósteos marinos, de agua dulce, salmónidos y especies vivíparas (Morisawa y col 1983, Cosson y col 1999, Darszon y col 1999).

La motilidad espermática de la perca es suprimida por altas osmolaridades (Lahnsteiner y col 1995). Un similar mecanismo de inhibición osmótica de motilidad espermática existe en ciprínidos (Morisawa 1983, citado por Lahnsteiner y col 1995) y en la lamprea marina, especie en que la motilidad espermática es inhibida por una osmolaridad alta $(80 \mathrm{mmol} \mathrm{NaCl}$ o $\mathrm{KCl})($ Cieresko y Dabrowski 2002). En salmónidos, Morisawa y col (1983) indican que la osmolaridad no es un factor determinante en la motilidad espermática.

Así también, espermatozoides de teleósteos marinos tales como "puffer fish", lenguado y de peces dulceacuícolas, como carpa, dorada, "zebrafish" y pejerrey son inmóviles en soluciones con o sin electrolitos cuando la osmolaridad es isotónica con el plasma seminal de $300 \mathrm{mOsmol} / \mathrm{kg}$. La motilidad sólo comienza cuando el semen es diluido en una solución hipotónica para el caso de peces de agua dulce y con una solución hipertónica para peces de agua salada (Morisawa 1994).

En "catfish" (Silurus glanisel), la presión osmótica del fluido seminal es de $280 \mathrm{mOsmol} / \mathrm{kg}$ y la activación ocurre en agua dulce o en soluciones salinas si la presión osmótica es más baja que $70 \mathrm{mOsmol} / \mathrm{kg}$ (Billard y col 1997).

En Galaxias maculatus la osmolaridad del fluido seminal alcanza a 299 mOsmol/kg (Fernández 2001, Muñoz 2001). McDowall (1968) y McDowall y col (1994) señalan que esta especie realiza su fecundación en ambientes estuarinos, por lo que es considerada como "catádroma marginal". Mitchell (1989) recomienda condiciones para la fecundación de $6 \mathrm{~g} / \mathrm{L}$ de salinidad (125 mOsmol/ kg aprox.). En cambio, Valdebenito (2004) obtiene la mayor motilidad y capacidad fecundante en agua dulce con cerca de $1 \mathrm{~m} 0 \mathrm{sm} / \mathrm{kg}$.

$\mathrm{pH}$

El pH intra o extracelular de teleósteos marinos y dulceacuícolas es reconocido como un importante regulador de la motilidad espermática (Márian y col 1997). Este factor en las soluciones activadoras de la motilidad espermática también afecta de distintas maneras la actividad flagelar del espermatozoide dependiendo de la especie (Cosson y col 1999, Woolsey y col 2006). El espermatozoide de la perca tiene un pH óptimo en el rango de 7,0 a 8,5 (Lahnsteiner y col 1995). En salmónidos y ciprínidos, el pH óptimo para la motilidad y capacidad fecundante es cercano a 9,0 (Billard 1989, Alavi y Cosson 2005) y la motilidad puede ser iniciada por la alcalinización (Billard y Cosson 1993, citado por Lahnsteiner y col 1995). En trucha arcoiris un pH bajo 7,8 no induce la motilidad espermática (Baynes y col 1981 citado por Cosson y col 1999). La aplicación de bicarbonato extracelular y alto $\mathrm{pH}$ para la adquisición de motilidad espermática en trucha arcoiris y salmón "chum", ha sido sugerida por Morisawa y col (1993).

El pH en el conducto seminal ( $\mathrm{pH} 8$ ) del salmón "chum" es más alto que en los testículos $(\mathrm{pH} 7,5)$, haciendo que adquiera mayor potencial de motilidad en el desplazamiento desde el testículo al conducto seminal (Morisawa 1994). En G. maculatus la mayor motilidad se obtiene con $\mathrm{pH}$ entre 5 y 7 (Vera 2004), presentando esta especie un $\mathrm{pH}$ en el fluido seminal de 7,96 (Muñoz 2001).

Espermatozoides de especies marinas como "mullet" (Mugil capito) son activados a pH entre 5,5 y 10,0, siendo el óptimo 7,0 (Hines y Yashouv 1971 citado por Cosson y col 1999). En el bacalao de profundidad, la motilidad espermática es activada a pH entre 5 y 10, siendo el óptimo alrededor de 7. Para el caso de la lamprea marina la duración de la motilidad espermática disminuye con los aumentos en el pH (Cieresko y Dabrowski 2002).

Woolsey y col (2006), trabajando con trucha arcoiris cabeza de acero, determinan un efecto significativo del pH de la solución de almacenamiento del semen en el nivel de motilidad y capacidad fecundante luego de la activación, estimando un $\mathrm{pH} 8,5$ como óptimo para el medio de almacenamiento.

\section{GASES}

\section{DIÓXIDO DE CARBONO}

Los niveles de $\mathrm{CO}_{2}$ en los medios de almacenamiento y activación de los espermatozoides de peces se relacionan en forma inversa con los valores del $\mathrm{pH}$ y de la osmolaridad producto de la formación de $\mathrm{HCO}_{3}^{-}$(Cosson y col 1999). La mantención de espermatozoides de salmónidos in vitro a concentraciones altas de $\mathrm{CO}_{2}$ inhibe la motilidad presumiblemente vía un efecto del $\mathrm{CO}_{2}$ en el $\mathrm{pH}$ (Bencic y col 2000). Por ejemplo, espermatozoides de trucha arcoiris almacenados a presiones de $\mathrm{CO}_{2}$ de $5,3 \mathrm{kPa}$ no se activan al ser diluidos en agua desionizada. Se activan muy poco en agua o fluido ovárico, pero demuestran una alta actividad flagelar al ser diluidos en un activador con pH 8,5 (Woolsey y col 2006).

\section{OXÍGENO}

Como regla general, en peces la producción de energía realizada en las mitocondrias de la pieza media del espermatozoide requiere de un flujo permanente de oxígeno (Cosson y col 1999), por lo tanto, la disponibilidad de este comburente es esencial para la capacidad de movimiento 
del espermatozoide, estimándose requerimientos de entre 20-40 $\mu \mathrm{l}$ de oxígeno por $10^{6}$ células por hora para especies como la trucha arcoiris, salmón del Atlántico y bacalao, los que son similares a los descritos para erizo e incluso humanos. Los requerimientos son máximos al inicio de la activación y disminuyen paulatinamente en la medida en que se reduce la actividad flagelar (Cosson y col 1999).

\section{COMPOSICIÓN IÓNICA}

Woolsey y col (2006) informan de la importante influencia de la composición iónica del medio externo sobre la motilidad espermática y cómo los canales iónicos son elementos esenciales para este proceso celular (Darszon y col 1999).

Como se señaló anteriormente, en salmónidos, la dilución del semen en soluciones con $\mathrm{K}^{+}$inhibe la motilidad espermática (Billard 1988, Cussac y Maggese 1988, Billard y col 1995a, Darszon y col 1999, Cosson y col 1999, He y Woods 2003, Woolsey y col 2006).

El efecto del $\mathrm{K}^{+}$en la motilidad espermática en otros peces teleósteos es menos claro, pero se ha determinado que no inhibe la motilidad flagelar en algunas especies (Morisawa 1994). En pez gato (Rhadmia sapo), la presencia de $\mathrm{K}^{+}$en distintas concentraciones no afecta la motilidad espermática (Cussac y Maggese 1988). De igual manera ocurre para especies de agua dulce como el pez dorado y "zebrafish" (Darszon y col 1999).

Por el contrario, la presencia de $\mathrm{K}^{+}$es necesaria para la iniciación de la motilidad espermática en la anguila japonesa (Ohta y col 1997). Cosson y col (1999) reportan que la presencia del ion $\mathrm{K}^{+}$en muy bajas concentraciones, en el rango de $0,01 \mathrm{mM}$, muestra un control sobre la activación espermática en "padle fish" y esturión (Acipenser baeri).

En revisión de Cosson y col (1999) se informa que iones monovalentes como $\mathrm{Na}^{+}$y bivalentes como $\mathrm{Ca}^{++}$, $\mathrm{Sr}^{++}, \mathrm{Ba}^{++} \mathrm{y} \mathrm{Mg}^{++}$reducen el efecto inhibidor del $\mathrm{K}^{+}$. Una relación $\mathrm{Na}^{+} / \mathrm{K}^{+}$de al menos $16: 1$ permite una adecuada actividad flagelar en especies salmonídeas (Alavi y Cosson 2006). En la actualidad, es conocido que la presencia de $\mathrm{Ca}^{++}$y otros segundos mensajeros como AMPc son importantes para la iniciación de la motilidad espermática en salmónidos al contrarrestar el efecto inhibidor del $\mathrm{K}^{+}$ (Billard y Cosson 1989, 1995, Darszon y col 1999, Cosson y col 1999). También en "padle fish" y esturión (Acipenser baeri) la presencia de $\mathrm{Ca}^{++}$es importante para la iniciación de la actividad espermática.

En contraste con lo observado en salmónidos, los espermatozoides de carpa presentan una independencia del ion $\mathrm{Ca}^{++}$, similar efecto es observado en varios peces teleósteos como "turbot", "halibut", silúridos y las anguilas (Cosson y col 1999).

Iones de metales pesados en el medio de dilución del semen muestran un deterioro en la motilidad espermática de animales marinos. Como por ejemplo, la presencia de
$\mathrm{Zn}^{++}$en bajas concentraciones en el agua de mar evita la activación del espermatozoide de "sea urchin". Los iones $\mathrm{Cd}^{++}$y $\mathrm{Zn}^{++}$afectan la motilidad del espermatozoide del pez gato. Los derivados del mercurio como $\mathrm{HgCl}_{2}$ afectan al espermatozoide de "turbot" y "sea bass". En puye no existe información ni antecedentes bibliográficos sobre cómo afecta la composición iónica del medio a la motilidad y capacidad fecundante del espermatozoide.

\section{TEMPERATURA}

La temperatura es otro factor importante que incide en el metabolismo de los espermatozoides de peces modelando los patrones de motilidad después de su activación en la gran mayoría de las especies. En general, bajas temperaturas prolongan la motilidad a través del tiempo y reducen la velocidad de desplazamiento (Billard y Cosson 1986, Vladic y Järvi 1997, Cosson y col 1999, Alavi y Cosson 2005).

Al evaluar el porcentaje de fecundación en puye se ha determinado que los mejores valores se obtienen a temperaturas cercanas a $10{ }^{\circ} \mathrm{C}$, temperaturas próximas a $15^{\circ} \mathrm{C}$ reducen su capacidad fecundante. Además, existe una correlación inversa entre el tiempo de motilidad del espermatozoide y la temperatura (Fletcher 2000), es decir, que a mayor temperatura la duración de la motilidad espermática es menor. En especies como el salmón del Atlántico y trucha marrón la fecundación puede ocurrir en rangos más amplios de temperatura $\left(2\right.$ a $\left.16^{\circ} \mathrm{C}\right)$ (Vladic y Järvi 1997). En "baltic cod" (Gadus morhua) la mayor motilidad espermática se registra a una temperatura de $7^{\circ} \mathrm{C}$. Esto demuestra que las temperaturas bajas benefician los patrones de motilidad en muchas especies.

Zuccarelli y col (2007) reportan que en burbot (Lota lota) la temperatura puede ser un factor iniciador de la motilidad espermática, ya que temperaturas superiores a $5{ }^{\circ} \mathrm{C}$ activan la motilidad espermática, a pesar de encontrarse los espermatozoides en plasma seminal.

\section{CAMBIOS MORFOLÓGICOS}

Producto del cambio sufrido por los espermatozoides después de ser diluidos en un medio hipotónico (en peces de agua dulce) e hipertónico (en peces de agua salada), la morfología celular cambia, hidratándose en el primer caso y deshidratándose en el segundo. Esto genera cambios en la capacidad de desplazamiento y si la dilución es prolongada puede llevar a la destrucción celular. Por ello la dilución de los espermatozoides en medios isotónicos reduce estos daños y prolonga el tiempo de motilidad espermática (Billard 1988, Cosson y col 1999).

\section{METALES PESADOS}

Los metales pesados ejercen un efecto negativo en la capacidad de movimiento de los espermato- 
zoides, aun a muy bajas concentraciones de algunos metales como el zinc, cadmio, cobre, nitrito, plomo y algunos derivados del mercurio (Cosson y col 1999, Lahnsteiner y col 2004). Este último autor determina que los espermatozoides del pez gato africano son los más resistentes a algunos de estos contaminantes. El burbot tiene una resistencia media y los de trucha café muestran la mayor sensibilidad. Van Look y Kime (2003) determinan que la exposición de espermatozoides de pez dorado a bajas concentraciones de mercurio altera la morfología del $98 \%$ de los gametos, incrementa el tamaño de la cabeza y reduce la longitud del flagelo y la duración de la motilidad.

\section{MANEJO DE GAMETOS EN PECES DE CULTIVO}

La preservación in vitro de gametos está asumiendo un rol importante en la producción de ovas y en el manejo genético de reproductores en pisciculturas. Las técnicas de almacenamiento por un periodo prolongado no ha dado una solución viable para el almacenamiento de ovas, no así para los espermatozoides, para los cuales existen varias formas de mantención que pueden ir desde la criopreservación hasta el almacenaje a bajas temperaturas (Bromage y Roberts 1995). La necesidad de mantener o preservar el semen de diversas especies de peces fuera del ambiente testicular, surge de:

- la asincronía en la madurez plena entre machos y hembras (McNiven y col 1993, Bromage y Roberts 1995),
- la necesidad de optimizar el manejo y eficiencia de un plantel reproductivo (Stoss y Holtz 1983, Christensen y Tiersch 1996),

- para estudios genéticos y preservación del patrimonio genético de especies en peligro de extinción (Stoss y Refstie 1983, Korokura y col 1986 en Christensen y Tiersch 1996),

- para almacenar el semen a la espera del diagnóstico ictiopatológico al que se somete a los reproductores para evitar la transmisión vertical de enfermedades, y

- para el traslado de gametos cuando los reproductores se encuentran distantes de los centros de cultivo en que se realizan la fecundación e incubación.

\section{DILUYENTES ESPERMÁTICOS}

Estas son soluciones salinas isotónicas cuyo fin es imitar el medio en que se encuentran los espermatozoides dentro del testículo, son también llamadas "extender, medio inmovilizante o dilutor". Son utilizados regularmente en el manejo artificial de gametos en mamíferos (Sánchez y Rubilar 2001). Los diluyentes para peces están basados principalmente en la composición química del plasma seminal de cada especie pues se deben mantener inmóviles los espermatozoides por periodos cortos de tiempo. También pueden ser agregados otros compuestos como algún tipo de buffer y glucosa, entre otros (Erdahl y Graham 1987). La composición química de estos compuestos es muy variada, incluyendo los recomendados para una misma especie. Por ello en el cuadro 3 se entregan ejemplos de algunos de los diluyentes utilizados en especies salmonídeas.

Cuadro 3. Diluyentes espermáticos utilizados para el almacenamiento de semen de salmónidos. Incubation media used to storage salmonid semen.

\begin{tabular}{|c|c|c|c|c|c|c|c|}
\hline Componente $(\mathrm{mM})$ & 1 & 2 & 3 & 4 & $5(\mathrm{~g} / \mathrm{L})$ & 6 & $7(\mathrm{~g} / 2 \mathrm{~L})$ \\
\hline $\mathrm{NaCl}$ & 80 & 80 & 110 & 103 & 5,16 & 110 & 11,7 \\
\hline $\mathrm{KCl}$ & 40 & 40 & 28,3 & 40 & 1,64 & 28,3 & 5,1 \\
\hline $\mathrm{CaCl} 2$ & 0,1 & 0,1 & - & 1,0 & 0,143 & 1,8 & 0,2 \\
\hline $\mathrm{NaHCO} 3$ & - & - & - & - & 1,00 & - & - \\
\hline $\mathrm{NaH} 2 \mathrm{PO} 4$ & - & - & - & - & 0,41 & - & - \\
\hline $\mathrm{Na} 2 \mathrm{HPO} 4$ & - & - & - & - & - & - & 0,5 \\
\hline Tris & 30 & 30 & 0,02 & 20 & - & 0,02 & - \\
\hline $\mathrm{MgSO} 4$ & - & - & 1,1 & - & 0,223 & 1,1 & - \\
\hline $\mathrm{MgSO} 2$ & - & - & - & 0,8 & - & - & - \\
\hline $\mathrm{MgCl} 2$ & - & - & - & - & - & - & 0,4 \\
\hline $\mathrm{CaCl}$ & - & - & 1,8 & - & - & - & - \\
\hline Fructosa & - & - & - & - & 1,00 & - & - \\
\hline Glucosa & - & - & - & - & - & - & 20 \\
\hline Ac. Cítrico & - & - & - & - & - & - & 0,2 \\
\hline Bicine $(5,3 \mathrm{~g} / 100 \mathrm{ml})$ & - & - & - & - & - & - & $20 \mathrm{ml}$ \\
\hline $\mathrm{KOH}(1,27 \mathrm{~g} / 100 \mathrm{ml})$ & - & - & - & - & - & - & $20 \mathrm{ml}$ \\
\hline $\mathrm{pH}$ & $9,26,5-8,5$ & 9,0 & 7,8 & 7,3 & 9 & 7,8 & \\
\hline Osmolaridad (mOsm) & - & - & - & - & 226 & - & 310 \\
\hline
\end{tabular}

1. Cosson y col 1999, 2. Woolsey y col 2006, 3. Billard 1983, 4. Lahnsteiner y col 2004, 5. Truscot y Idler 1969, 6. Billard y Jalabert 1974, 7. Erdahl y Gram 1987. 
Algunos investigadores han estudiado el uso de un diluyente que imita el contenido del plasma seminal de peces de importancia comercial, principalmente truchas y salmones. Truscott e Idler (1969) en Erdahl y Graham (1987) reportan motilidad de los espermatozoides de salmón del Atlántico después de 6 días de almacenamiento con un diluyente que contenía principalmente cloruro de sodio, de potasio y calcio, fructosa, agua y $\mathrm{pH} 7,3$. Erdahl y Graham (1987) almacenaron semen de trucha por $24 \mathrm{hr}$ usando un diluyente cuyos componentes principales eran cloruro de sodio, de potasio, de magnesio y glucosa, con buenos resultados de fecundación. En revisión realizada por Linhart y col (1995) se informa del almacenamiento de semen de Polyodon spathula por periodos cortos de tiempo usando una solución salina de cloruro de sodio al 0,9\%. Conte y col (1998) en Linhart y col (1995) almacenaron semen de esturión blanco (Acipenser transmuntanus) por 14 días a $4{ }^{\circ} \mathrm{C}$. Bromage y Roberts (1995) mencionan que suministrar oxígeno y/o la adición de antibióticos junto con bajas temperaturas de almacenamiento prolongan el periodo viable de los espermatozoides. Similares resultados fueron determinados por Valdebenito (2004) para el almacenamiento de semen de puye durante tres días sin perder significativamente la capacidad fecundante.

Trabajos con semen de trucha arcoiris demostraron la conveniencia de la combinación de penicilina y estreptomicina para almacenamiento de semen durante tiempos prolongados (Stoss y Holtz 1983). Los antibióticos son comúnmente usados para proteger semen de mamíferos durante el almacenaje, pero han sido poco utilizados en salmónidos.

McNiven y col (1993) diluyeron semen de trucha arcoiris en medios no acuosos con fluorocarbonos almacenando semen hasta por 37 días con aceptables niveles de motilidad.

Mientras el semen se encuentra en un diluyente espermático, se deben resguardar la temperatura y luminosidad. Regularmente, el almacenamiento se realiza a oscuras y a temperaturas de entre $0-4{ }^{\circ} \mathrm{C}$, idealmente (Billard 1983, Stoss y Refstie 1983, Gordon y col 1987, Vladic y Järvi 1997, Lahnsteiner y col 2004). Frecuentemente, se inyecta oxígeno gaseoso para prolongar aún más la sobrevivencia y viabilidad de los espermatozoides. Gordon y col (1987) recomiendan una relación de 1:120 entre el volumen de semen almacenado y del gas que lo cubre. Además, indican que la altura de la columna de semen no debe superar los $6 \mathrm{~mm}$. Esto permite almacenar semen por varios días con buena viabilidad. Con frecuencia, el semen se diluye en una relación 1:2 con respecto del diluyente espermático. Durante el almacenamiento, el pH del medio es un factor importante que determina la capacidad de movimiento del espermatozoide luego de la activación. Woolsey y col (2006) determinan que el semen de trucha arcoiris almacenado por dos horas a pH 8,5 presenta mayor porcentaje de espermatozoides mótiles que el semen almacenado a $\mathrm{pH} 7,1$.

El uso de diluyentes espermáticos resuelve de alguna manera el problema de la mantención del semen fuera del testículo, permitiendo aumentar el período en que los espermatozoides mantienen su capacidad fecundante, lo que significa una gran ayuda para los productores de ovas. Esto es evidente en pisciculturas de salmónidos, en donde se necesita preservar los gametos por varios días en espera de los resultados del "screening" realizado a cada uno de los reproductores, o transportar los gametos por varias horas cuando los reproductores se encuentran lejos de los "hatcheries".

\section{ACTIVADORES ESPERMÁTICOS}

Existen varios trabajos en que se ha sometido al semen de peces a diversos ambientes extratesticulares para poder determinar los factores que controlan su activación e inhibición, viabilidad y comportamiento. Algunas soluciones utilizadas en estas investigaciones se podrían clasificar como "soluciones activadoras" ya que tratan de prolongar el tiempo e intensidad de la motilidad del espermatozoide y, con ello, su capacidad fecundante. En salmónidos, el fluido ovárico o líquido celómico que acompaña las ovas durante el desove es un activador natural muy efectivo para el espermatozoide (Billard 1983, Woolsey y col 2006), por lo que muchos activadores espermáticos utilizados en estas especies se basan en imitar su composición (cuadro 4).

Cuadro 4. Activadores espermáticos utilizados para la activación de la motilidad espermática en salmónidos. Activation media used to trigger the sperm motility in salmonids.

\begin{tabular}{|c|c|c|c|}
\hline Componente (mM) & 1 & 2 & 3 \\
\hline $\mathrm{NaCl}$ & 125 & 125 & 155 \\
\hline $\mathrm{KCl}$ & 0 & 0 & 3,1 \\
\hline $\mathrm{CaCl} 2$ & 0,1 & 0,1 & - \\
\hline Tris & 30 & 30 & 0,02 \\
\hline $\mathrm{MgSO} 4$ & - & - & 1,3 \\
\hline $\mathrm{CaCl}$ & - & - & 3,4 \\
\hline $\mathrm{pH}$ & 9,2 & 8,5 & 9,0 \\
\hline Otros & - & 1,0 & - \\
\hline
\end{tabular}

1. Cosson y col 1999, 2. Woolsey y col 2006, 3. Billard 1983. 
Según aparece en Erdahl y Graham (1987), Poon y Johnson (1970) estudiaron la activación del semen de teleósteos para incrementar el número de ovas que pueden ser fecundadas usando agua dulce con pobres resultados. Valdebenito (2007) evaluó el efecto de una solución activadora que incluye cafeína sobre la motilidad del espermatozoide de trucha arcoiris, encontrando que se incrementaron los niveles de motilidad en forma significativa respecto del control (agua de río) al usar una concentración de 3,5 mM de cafeína. Las soluciones salinas también han sido largamente estudiadas como un posible medio de activación y mostraron resultados más favorables que con agua dulce, pero con tiempos de motilidad más limitados (Erdahl y Graham 1986). Goodal y col (1989) examinaron algunos de los factores que regulan la activación y duración de la motilidad del semen de Sillago ciliata orientados a la capacidad de fecundación de los espermatozoides usando soluciones que contenían cloruro de sodio, de potasio y glucosa a distintas osmolaridades, encontrando que al usar medios de activación que contengan cloruro de sodio y de potasio a osmolaridades de $600 \mathrm{mOsm}$ se incrementa la motilidad de los espermatozoides. Ohta y col (1997) utilizaron soluciones salinas basadas en los componentes iónicos del plasma seminal de Anguilla japonica, modificando las concentraciones de algunos componentes como potasio, bicarbonato y $\mathrm{pH}$ para determinar cuáles son los factores que promueven la motilidad en esta especie. Encontraron que al elevar las concentraciones de potasio, bicarbonato y el $\mathrm{pH}$ de las soluciones en un rango de 7,8 a 8,7 se incrementa el porcentaje de espermatozoides mótiles.

Productivamente, las pisciculturas de especies salmonídeas instaladas en Chile (observación personal) regularmente utilizan soluciones salinas o activadores espermáticos en una proporción cercana al $20-30 \%$ del volumen de las ovas para realizar la fecundación. Con esto, se prolonga la duración de la motilidad espermática, la intensidad del movimiento flagelar y, finalmente, la capacidad fecundante del semen.

\section{CONCLUSIONES}

El conocimiento de los patrones de motilidad espermática en peces de cultivo es una herramienta clave para la optimización del uso del semen en las actividades de piscicultura con el fin de obtener altos porcentajes de fecundación. Los factores más importantes que regulan la actividad flagelar en espermatozoides de peces de fecundación externa son la osmolaridad y la composición iónica del medio, con efectos específicos para cada especie. En peces de agua dulce, un medio hipotónico inicia la actividad flagelar. En cambio en peces marinos lo hace un medio hipertónico. En salmónidos, por ejemplo, niveles altos de $\mathrm{K}^{+}$bloquean la motilidad espermática y la presencia de $\mathrm{Ca}^{++}$es esencial para su activación. Previo a la activación del espermatozoide, temperaturas bajas $\left(2^{\circ} \mathrm{C}\right.$ aproximada- mente) y niveles altos de oxígeno prolongan la viabilidad del espermatozoide.

La osmolaridad y composición iónica de los medios de activación son factores claves para determinar la capacidad de almacenamiento del semen. Por otra parte, factores como la temperatura y $\mathrm{pH}$ juegan un rol clave en la modelación del patrón de motilidad espermática. Temperaturas bajas permiten prolongar la actividad espermática, aunque el movimiento flagelar es menos intenso. Los diluyentes y activadores espermáticos surgen como herramientas útiles para optimizar el manejo in vitro del semen de peces en cultivo frente a la necesidad de almacenarlo en forma prolongada, o bien transportarlo hacia los centros de cultivo en los cuales se realiza el proceso de fecundación e incubación.

\section{RESUMEN}

En la mayoría de los peces teleósteos de fecundación externa los espermatozoides se encuentran inmóviles en el fluido seminal y son activados sólo después de tomar contacto con un medio acuoso. El tiempo de actividad flagelar es breve en la mayoría de las especies. Los factores que activan este proceso son diferentes para cada especie. Sin embargo, en casi todas, factores como la osmolaridad, temperatura, $\mathrm{pH}$ y composición iónica tienen una función clave en activar o modelar la actividad flagelar. La presente revisión analiza los factores que participan en la activación y regulación de la activación espermática en peces de fecundación externa. Además, se realiza un análisis respecto del manejo en cultivo del semen de peces de interés comercial, con énfasis en especies salmonídeas.

\section{AGRADECIMIENTOS}

Se agradece el apoyo del proyecto FONDEF D06I1020 y a la Dirección de Investigación de la Universidad Católica de Temuco a través del proyecto DGIUCTemuco N² 2007-DGI-CDA-05. Además, al Dr. Adrián Hernández por la elaboración del resumen en inglés.

\section{REFERENCIAS}

Aas GH, T Refsti, B Gjerde. 1991. Evaluation of milt quality of Atlantic salmon. Aquaculture 95, 125-132.

Alavi SM, J Cosson. 2005. Sperm motility in fishes. I. Effects of temperature and pH: a review. Cell Biol Int 29, 101-110.

Alavi SM, J Cosson. 2006. Sperm motility in fishes. II. Effects of ions and osmolality: a review. Cell Biol Int 30, 101-110.

Bencic DC, JG Cloud, RL Ingermann. 2000. Carbon dioxide reversibly inhibits sperm motility and fertilizing ability in steelhead (Oncorhynchus mykiss). Fish Physiol Biochem 23, 275-281.

Billard R. 1978. Changes in structure and fertilizing ability of marine and freshwater fish spermatozoa diluted in media of various salinities. Aquaculture 14, 187-198.

Billard R. 1983. Effects of coelomics and seminal fluids and varius saline diluents on the fertilizing ability of spermatozoa in the rainbow trout, Salmo gaidneri. J Reprod Fert 68, 77-84.

Billard R. 1984. La conservation des gametes et l'insemination artificielle chez le bar et la daurade. In: Barnabé G, Billard R (ed). L'aquaculture $d u$ bar et des sparides. INRA Publ, Paris, France, Pp 95-116.

Billard R, MP Cosson. 1986. Sperm motility in rainbow trout, Parasalmo mykiss; effects of $\mathrm{pH}$ and temperature. Reprod Fish 44, 10-12.

Billard R. 1988. Artificial insemination and gamete management in fish. Mar Behav Phisiol 14, 3-21. 
Billard R, MP Cosson. 1989. Measurement of sperm motility in trout and carp. Aquaculture 132, 499-503.

Billard R, MP Cosson. 1990. The energetics of fish sperm motility. In: Gagnon C (ed). Controls of sperm motility: biological and clinical aspects. CRS Press, Quebec, Canada, Pp 153-173.

Billard R. $1990^{\mathrm{a}}$. Artificial insemination in fish. Marshall's Physiology of Reproduction 9, 870-889.

Billard R. 1990 . La motilité du spermatozoide de poisson: aspects énergétiques. $5^{a}$ Jornadas Internacionales de Reproducción Animal. Zaragoza, España.

Billard R, J Cosson, L Crim. 1993. Motility of fresh and aged halibut sperm. Aquat Living Resour 6, 67-75.

Billard R, J Cosson, LW Crim, M Suquet. 1995a. Sperm physiology and quality. In: Bromage N, Roberts RJ (eds). Broodstock Management and egg and larval quality. Blackwell Sciences Ltd, Cambridge, UK, Pp 25-52.

Billard R, J Cosson, G Perchec, O Linhart. 1995 b. Biology of sperm and artificial reproduction in carp. Aquaculture 129, 95-112.

Billard R, O Linhart, F Fierville, J Cosson. 1997. Motility of European catfish Silurus glanis spermatozoa in testes and milt. Polskie Archiwum Hidrobiology 44, 115-122.

Bromage N, R Roberts. 1995. Broodstock management and egg and larval quality. Blackwell Science, Oxford, UK.

Chereguini O, B Peleteiro, R Cal, A García. 1995. Preliminary results of different methods of artificial fertilization with fresh sperm of turbot (Scophthalmus maximus L.) ICES Mar Sci Symp 201, 170-172.

Christensen JM, TR Tiersch. 1996. Refrigerated storage of Channel Catfish sperm. $J$ Word Aquacult Soc 27, 340-343.

Ciereszko A, GP Toth, SA Christ, K Dabrowski. 1996. Effect of cryopreservation and theophylline on motility characteristics of lake sturgeon (Acipenser fulvescens) spermatozoa. Theriogenology 45, 665-672.

Ciereszko A, K Dabrowski. 2002. Factors affecting motility characteristics and fertilizing ability of sea lamprey spermatozoa. T Am Fish Soc 131, 193-202.

Cosson J, R Billard, C Cibert, C Dreanno, M Suquet. 1999. Ionic factors regulating the motility of fish sperm. In: Gagnon $\mathrm{C}(\mathrm{ed})$. The Male Gamete: From basic knowledge to clinical applications. Cache River Press, Paris, France, Pp 161-186.

Cussac V, M Maggese. 1988. Effects of salt solutions on the fertilizing ability and activation of gametes in the catfish, Rhadmia sapo, (pisces, pimelolidae). Rev Brasil Biol 48, 203-211.

Darszon A, P Labarca, N Takuya, F Espinoza. 1999. Ion channels in sperm physiology. Physiol Rev 79, 486-502.

Elofsson H, BG Mcallister, DE Kime, I Mayer, B Borg. 2003. Long lasting stickleback sperm; is ovarian fluid a key to success in fresh water? J Fish Biol 63, 240-253.

Erdahl AW, EF Graham. 1987. Fertility of teleost semen as affected by dilution and storage in a seminal plasma-mimicking medium. Aquaculture 60, 311-321.

Estay F, H Cerisola, V Téllez. 1994. Biología del desarrollo y reproducción artificial en la trucha arcoiris. CONICYT-FONDEF, Serie Publicaciones para la acuicultura $\mathrm{N}^{\circ} 1$.

Fernández J. 2001. Evaluación de técnicas de almacenamiento de semen en puye (Galaxias maculatus) y salmón coho (Oncorhynchus kisutch) a bajas temperaturas utilizando distintos medios acuosos para su dilución: su efecto en la fertilidad. Memoria de título, Escuela de Acuicultura, Universidad Católica de Temuco, Temuco, Chile.

Fletcher del Castillo C. 2000. Estudio de los patrones de motilidad y fertilidad del espermatozoide de puye (Galaxias maculatus) (Jenyns, 1842) bajo condiciones de cultivo experimental. Memoria de título, Escuela de Acuicultura, Universidad Católica de Temuco, Temuco, Chile.

Ginzburg A. 1972. Fertilization in fishes and the problem of polispermy. Academy of Sciences of the URSS, Moscu, URSS

Goodall J, A Blackshaw, M Capra. 1989. Factors affecting the activation and duration of motility of the spermatozoa of the summer whiting (Sillago ciliata). Aquaculture 77, 243-250.
Gordon MR, KC Klotins, VM Campbell, MM Cooper. 1987. Farmer salmon broodstock management. B.C. Research, Vancouver, B.B., Canada.

He S, C Woods. 2003. The effects of osmolality, cryoprotectant and equilibration time on striped bass Morone saxatilis sperm motility. $J$ Word Aquacult Soc 34, 255-265.

Hoysak DJ, NR Liley. 2001. Fertilization dynamics in sockeye salmon and a comparison of sperm from alternative male phenotypes. J Fish Biol 58, 1286-1300.

Krasznai Z, T Márian, L Balkay, R Gáspár, L Trón. 1995. Potassium channels regulate hypo-osmotic shock-induced motility of common carp (Cyprinus carpio) sperm. Aquaculture 129, 123-128.

Lahnsteiner F, R Patzner, T Weismann. 1993. Energy resources of spermatozoa of the rainbow trout Oncorhynchus mykiss. (Pisces, teleostei). Reprod Nutr Dev 33, 349-360.

Lahnsteiner F, B Berger, W Teismann, R Patzner. 1995. Fine structure and motility of spermatozoa and composition of the seminal plasma in the perch. J Fish Biol 47, 492-508.

Lahnsteiner F, N Mansour, B Berger. 2004. The effect of inorganic and organic pollutants on sperm motility of some freshwater teleosts. $J$ Fish Biol 65, 1283-1297.

Levanduski MJ, JG Cloud. 1988. Rainbow trout (Salmo gairdneri) semen: Effect of non-motile sperm on fertility. Aquaculture 75, 171-179.

Linhart, O, D Mims, L Shelton. 1995. Motility of spermatozoa from shovelnose sturgeon and paddlefish. J Fish Biol 47, 902-909.

Márian T, Z Krasznai, L Balkay, M Emri, L Trón. 1997. Role of extraand intracellular $\mathrm{pH}$ in the sperm motility. Hyperosmosis modifies regulation of the $\mathrm{Na}^{+} / \mathrm{H}^{+}$exchanger. Cytometry $27,374-382$.

McDowall R. 1968. Galaxias maculatus (Jenyns), the New Zealand Whitebait. Fish Res Bulletin 2, 9-84.

McDowall R, C Mitchell, Brothers E. 1994. Age at migration from the sea of juvenile galaxias in New Zealand (Pisces: Galaxiidae). Mar Sci 54, 385-402.

McNiven MA, RK Gallant, GF Richardson. 1993. Fresh storage of rainbow trout (Oncorhynchus mykiss) semen using a non-aqueosus medium. Aquaculture 109, 71-82.

Mongkonpunya K, T Pupipat, A Hambananda, N Chairak, T Tiersch. 1996. Effect of osmotic pressure on sperm motility of mekong giant catfish and chao phraya catfish. Thai J Agric Sci 29, 581-588.

Morisawa M, K Suzuki. 1980. Osmolality and potassium ion: their roles in initiation of sperm motility in teleost. Science 210, 1145-1147.

Morisawa M, K Suzuki, S Morisawa. 1983. Effects of potassium an osmolality on spermatozoan motility of salmonids fishes. J Exp Biol 107, 105-113.

Morisawa M. 1994. Cell signalling mechanism for sperm motility. Zool Sci 11, 647-662.

MuñozA. 2001. Bioensayos preliminares para la criopreservación de semen de puye Galaxias maculatus (Jenyns, 1842) (Pisces: Salmoniformes). Memoria de título, Escuela de Acuicultura, Universidad Católica de Temuco, Temuco, Chile.

Ohta H, H Tanaka, H Kagawa, K Okuzawa, N Iinuma. 1997. Artificial fertilization using testicular spermatozoa in the japanese eel Anguilla japonica. Fisheries Sci 63, 393-396.

Paniagua-Chávez C, J Buchanan, T Tiersch. 1998. Effect of extender solutions and dilution on motility and fertilizing ability of eastern oyster sperm. J Shellfish Res 17, 231-237.

Redondo-Müller C, M.P Cosson, J Cosson, R Billard. 1991. In vitro maturation of the potential for movement of carp spermatozoa. $\mathrm{Mol}$ Reprod Dev 29, 259-270.

Rudolfsen G, L Figenschou, I Folstad, O Kleven. 2008. Sperm velocity influence paternity in the Atlantic cod (Gadus morhua L.). Aquaculture Research 39, 212-216.

Sánchez-Rodríguez M, R Billard. 1977. Conservation de la motilité et du puvoir fécondant du sperm de truite arc en ciel maintenu á des temperatures voisines de $0^{\circ} \mathrm{C}$. Bulletin Français de Pisciculture 265, 143-152.

Sánchez A, J Rubilar. 2001. Obtención de cachorros mediante inseminación artificial con semen canino refrigerado: Primera descripción en Chile. Arch Med Vet 33, 105-110. 
Scott A, S Baynes. 1980. A review of the biology handling and storage of salmonid spermatozoa. J Fish Biol 17, 707-739.

Stoss J, W Holtz. 1983. Successful storage of chilled rainboi trout (Salmo gairdneri) spermatozoa for up to 34 days. Aquaculture 31, 269-274.

Stoss J, T Refstie. 1983. Short- term storage and cryopreservation of milt from Atlantic Salmon and Sea Trout. Aquaculture 30, 229-236.

Strüssmann C, P Renard, H Ling, F Takashima. 1994. Motility of pejerrey Odontesthes bonariensis spermatozoa. Fis Sci 60, 9-13.

Terner Ch. 1986. Evaluation of salmonid sperm motility for cryopreservation. The Progressive Fish-Culturist 48, 230-232.

Trippel E, D Perley, J Neilson. 1991. Spermatocrit and swimming speed do not correlate with fertilization success in Atlantic cod (Gadus morhua). In: Lavens P, Sorgeloos P, Jaspers E, Ollevier F (eds). LARVI'91-Fish \& Crustacean Larviculture Symposium. European Aquaculture Society, Special Publication No 15, Gent, Belgium.

Truscott B, DR Idler. 1969. An improved extender for freezing Atlantic salmon spermatozoa. J Fish Res Board Can 26, 3254-3258.

Valdebenito I. 2003. Estudio de los parámetros reproductivos del puye (Galaxias maculatus, Jenyns, 1842) (Osmeriformes: Galaxiidae) bajo condiciones de cultivo experimental en el Sur de Chile. Tesis Doctoral. Universidad de Las Palmas de Gran Canaria, España.

Valdebenito NI. 2007. Efecto de la cafeína en la motilidad y fertilidad espermática de trucha arcoiris (Oncorhynchus mykiss). Información Tecnológica 18, 61-65.
Van Look KJW, DE Kime. 2003. Automated sperm morphology analysis in fishes: the effect of mercury on goldfish sperm. J Fish Biol 63, 1020-1033.

Vladic T, T Järvi. 1997. Sperm motility and fertilization time span in Atlantic salmon and brown trout-the effect of water temperature. J Fish Biol 50, 1088-1093.

Wayman WR, RG Thomas, TR Tiersch. 1996. Cryopreservation of sperm of seatrout (Cynoscion nebulosus). Gulf Research Reports 3, 183-188.

Wayman WR, TR Tiersch. 1998. Refrigerated storage and cryopreservation of sperm of red drum, Sciaenops ocellatus L. Aquac Res 29, 267-273.

Woolsey J, M Holcomb, J Cloud, R Ingermann. 2006. Sperm motility in the steelhead Oncorhynchus mykiss (Walbaum): influence of the composition of the incubation and activation media. Aquac Res 37, 215-223.

Zavos PM, JR Correa, PN Zarmakoupis-Zavos. 1996. Measurement of the sperm motility index via the sperm quality analyzer and its relationship to other qualitative sperm parameters. Theriogenology 13, 420-427.

Zuccarelli MD, N Jensen, RL Ingermann. 2007. Inhibitory effect of osmotic concentration, potassium and $\mathrm{pH}$ on motility of the sperm of the North American burbot Lota lota maculosa. J Fish Biol 70, 178-189. 